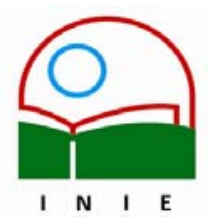

Universidad de Costa Rica

Facultad de Educación

Instituto de Investigación en Educación

ACTUALIDADES INVESTIGATIVAS EN EDUCACION

\title{
CARACTERIZACIÓN DE UN PROCESO DE AUTOEVALUACIÓN. DE LO TÉCNICO A LO INVESTIGATIVO.
}

\begin{abstract}
Silvia Chacón Ramírez ${ }^{1}$
Resumen: Este artículo es el segundo de una secuencia, cuya intención es abordar el tema de la autoevaluación. Este tópico es llevado al plano de la investigación, con el propósito de lograr una comprensión de lo que se realiza, de acuerdo con las intenciones de la autoevaluación, en el entendido de que éstas pueden variar, entre el control externo y la búsqueda del bien común. Esta última se encuentra, por estatuto, establecida en la Universidad de Costa Rica. El contexto para este artículo se ubica en el proceso de autoevaluación desarrollado en la Escuela de Formación Docente de la Facultad de Educación, de la Universidad de Costa Rica. En el primer artículo (Revista Electrónica. INIE. Vol. 5, N. 1, 2005) se ofrecían algunas premisas y cuestionamientos que develaban, en alguna medida, lo ideológico y epistemológico de un proceso de autoevaluación. En este segundo artículo, se presenta una "caracterización del modelo investigativo" ejecutado en el proceso de autoevaluación de la Escuela de Formación Docente, entre el año 2000 y el 2002.

Esta presentación incluye una identificación e interpretación de las características paradigmáticas investigativas (positivistas y naturalistas) del proceso autoevaluativo, una identificación de las premisas investigativas que dan sustento, la determinación de la intencionalidad de la autoevaluación, el papel desempeñado, tanto por el equipo investigador, como por los participantes. Adicionalmente se explican las características y fases del proceso metodológico. La conclusión hace referencia a los logros obtenidos, las limitaciones, para finalmente señalar lo que podría llegar a ser la autoevaluación en un contexto pertinente.
\end{abstract}

Palabras clave: INVESTIGACIÓN EVALUATIVAV AUTOEVALUACIÓN/ MODELO INVESTIGATIVO/ PARADIGMAS INVESTIGATIVOS/

Abstract: This is the second article of a sequence, which intends to tackle auto evaluation issues. This topic is brought to the research field to comprehend what is being done, considering that it purposes may vary between external control and common welfare lawfully established at the Universidad de Costa Rica. This article is within the context of the auto evaluation process developed at the Teacher Education School at the Faculty of Education. In the first article (Electronic journal INIE. Vol. 5, N. 1, 2005) some questions and premises that partially revealed the idelogic and epistemological aspects of an auto evaluation process were discussed. In this second article a "research model's characterization" is presented for the auto evaluation process executed at the Teachers and Lectures Formation School Teachers Education School during the 2000 to 2002 period.

This research model's characterization includes the identification of research paradigmatic characteristics (positivist and naturalistic) an interpretation of these characteristics, the identification of research premises, determination of auto evaluation intentionality, research team role, participant role, some characteristics and phases of the methodological process. Conclusions refere to goals, constraints and auto evaluation expectations within a pertinent context.

Key words: EVALUATION RESERCH/ AUTOEVALUATION/ RESERCH MODEL/RESERCH PARADIGMS/

\begin{abstract}
${ }^{1}$ Maestría Profesional en Planificación Curricular y Licenciatura en Educación Preescolar ambos títulos de la Universidad de Costa Rica. Profesora de la Escuela de Formación Docente de la misma universidad en los campos de Educación Preescolar, principalmente en las áreas de Artes del Lenguaje, Experiencia Profesional Docente, Literatura Infantil, Desarrollo de la Creatividad, Investigación. La labor investigativa más reciente se ubica en los campos de autoevaluación y la Educación Ambiental.
\end{abstract}

Correo electrónico: schacon@cariari.ucr.ac.cr

Artículo recibido: 14 de julio, 2005

Aprobado: 19 de setiembre, 2005 


\section{Introducción}

En este artículo se presenta, la caracterización metodológica del proceso de autoevaluación en la Escuela de Formación Docente de la Universidad de Costa Rica, como proceso de investigación evaluativa, con el propósito de dotar a la comunidad universitaria y aquellas personas interesadas, de un cuerpo de reflexión tal, que pueda hacer trascender al proceso de autoevaluación, del plano meramente técnico al plano de lo investigativoevaluativo.

Ahora bien, para comprender la transición de lo técnico a lo investigativo, se ofrece una caracterización del modelo utilizado. Por consiguiente, se incluyen los siguientes componentes:

- Contexto de la experiencia de autoevaluación.

- Dos objetos de estudio

- Condiciones que transforman la autoevaluación en un proceso de investigación.

- Caracterización de un modelo de autoevaluación: un modelo fluctuante.

\section{Contexto de la experiencia.}

El proceso investigativo abarcó un período de tres años y medio (1999-2002), cuyos propósitos fundamentales fueron:

- El logro de insumos para el mejoramiento en el desempeño de los planes de formación de docentes de Educación Preescolar, Educación Primaria y Educación Secundaria (Núcleo Pedagógico) ${ }^{2}$, en sus niveles de bachillerato, ofrecidos por la Escuela de Formación Docente de la Universidad de Costa Rica;

- Optar por la acreditación ante el Sistema Nacional de Acreditación (SINAES), de los planes de Educación Preescolar y Educación Primaria ${ }^{3}$.

- Brindar retroalimentación para la construcción reflexiva de un modelo de autoevaluación.

En este marco de objetivos, la búsqueda de un sustento teórico para el proceso de autoevaluación, tuvo su origen en 1999, cuyo interés se mantuvo durante todo el proceso.

${ }^{2}$ El Núcleo Pedagógico se constituye de un bloque de cursos comunes a 10 planes de estudio orientados a la enseñanza en Educación Secundaria: Inglés, Francés, Estudios Sociales, Matemática, Ciencias Naturales, Castellano y Literatura, Psicología, Filosofía, Música, Artes Plásticas.

${ }^{3}$ La acreditación constituye un acto en el que una instancia acreditadora da fe pública de la calidad de los programas o carreras, en el caso universitario, posterior a un proceso de autoevaluación de dichas carreras. 
De tal manera, a partir del segundo ciclo del mismo año, la señora directora de la Escuela de Formación Docente, en ejercicio, integró una comisión de autoevaluación con las directoras de los departamentos de Educación Secundaria, Docencia Universitaria, Educación Primaria y Preescolar, específicamente las profesoras, Carmen Liddy Fallas, Ana Cecilia Hernández y Silvia Chacón, quien suscribe y le correspondió asumir un doble papel; por una parte ser la coordinadora de comisión de autoevaluación y por otro, cumplir como investigadora principal.

El propósito de dicha comisión fue organizar y ejecutar el proyecto de autoevaluación, como respuesta al mandato de la Asamblea de Escuela. Para tal efecto, se inscribió un proyecto de investigación en el Instituto de Investigaciones para el Mejoramiento de la Educación Costarricense (IIMEC), hoy Instituto Nacional de Investigación en Educación (INIE), con el fin de mantener un rigor académico en el proceso.

\section{Dos objetos de estudio}

Se determinaron dos objetos de estudio que se atendieron simultáneamente: el primero lo constituyó el estado de desarrollo y operacionalización de las carreras de Educación Preescolar, Educación Preescolar y el Núcleo Pedagógico de Secundaria, respecto a la Unidad Académica. Para tal efecto, se utilizó como insumo operativo principal, la Guía de Autoevaluación, en su versión de 1998, facilitada por el Centro de Evaluación Académica (CEA) de la Universidad de Costa Rica, la cual considera seis factores:

- Plan de estudios, en este caso los Bachilleratos en Educación Primaria, Educación Secundaria y el tramo de carrera "Núcleo Pedagógico para las Enseñanzas en secundaria.

- La docencia

- El personal académico

- La población estudiantil

- La organización y administración

- Los recursos financieros, físicos y materiales

Por otra parte, el objetivo número tres de la investigación, esto es, retroalimentar con insumos para la constitución de un modelo de autoevaluación, configuró al "proceso de autoevaluación" como un segundo objeto de estudio. Por ello el desarrollo del proceso de autoevaluación y su reflexión se constituyeron en estrategias fundamentales. 


\section{Condiciones que transforman la "autoevaluación" en un proceso de investigación.}

Ya en el primer artículo "Acercamiento paradigmático de la autoevaluación como investigación" se anotan, con mayor precisión, las condiciones que transforman la autoevaluación en un proceso de investigación, sin embargo, para ubicar al lector en el tema, se resumen a continuación, algunas de las características principales:

a) En el entendido de que la autoevaluación es una evaluación, entonces se comprende que:

- La investigación evaluativa cuenta con un marco de referencia que la conduce al plano de la conciencia de su razón de ser. Se ubica dentro de una posición paradigmática para entender y sustentar varios planos: los resultados, el procedimiento, las consecuencias y las proposiciones.

- La evaluación plantea "valoraciones fundamentadas". La tarea básica de la investigación es "la producción de nuevos conocimientos" (Chacón, 2005, p.12).

- En el caso de que la evaluación conduzca al interjuego entre comprensión y valoración, ocurre una actividad de investigación, en la medida en que al realizar un análisis conceptual del objeto por evaluar y al construir una síntesis, tanto conceptual como teórica sobre él mismo, se está produciendo un conocimiento nuevo sobre tal objeto evaluado, según como lo señala de Alba (1991). Ahora bien, ese conocimiento puede ser de muy diversa índole.

- Concretamente, se puede concebir a la evaluación como una investigación evaluativa, en la medida en que para toda tarea de evaluación, se conjugan aspectos teóricos (vinculados con la producción de conocimiento) y aspectos axiológicos (vinculados con la valoración y el compromiso) (Chacón, 2005).

b) En un proceso de autoevaluación, las personas responsables tienen un alto grado de compromiso ante la situación, se ven involucradas y afectadas por el proceso y los resultados, es decir, se ven implicadas.

c) La autoevaluación como investigación evaluativa es propositiva. Necesariamente establece propuestas pertinentes a una realidad específica, para lograr una mejor condición.

d) Institucionalmente el proceso de autoevaluación en la Escuela de Formación Docente, fue un proceso evaluativo más; sin embargo, desde el punto de vista de 
su equipo investigador, fue un proceso llevado al plano de la investigación, por cuanto, no sólo se planteó el alcance de resultados y propuestas de autorregulación con miras a la acreditación, sino que se buscó la comprensión de cómo se realizó la investigación, además del esclarecimiento de su posición paradigmática, para trascender hacia una cultura de mirarse a sí mismos y alcanzar una mejor conformación de lo propuesto como imagen en su Proyecto Pedagógico. Particularmente cuatro aspectos evidencian el plano investigativo del proceso de autoevaluación desarrollado:

- El acercamiento desde el punto de vista paradigmático de la investigación.

- La sistematización, tanto metodológica, como de resultados.

- La develación de reflexiones producidas por los sujetos involucrados y el equipo responsable.

- La caracterización del modelo de autoevaluación desarrollado, más allá de la simple indicación de factores, criterios e indicadores por tomar en cuenta.

\section{Enfoques de la evaluación: un punto de vista evolutivo}

Esta perspectiva la presentan Guba y Lincoln (1989), quienes distinguen cuatro generaciones de cambio en los procesos evaluativos. La primera se encuentra centrada en el "rendimiento escolar", la segunda "descriptiva", la tercera "de juicio" y la cuarta "constructiva". A continuación se explican brevemente y se establece una relación con la autoevaluación.

- La primera generación utiliza el rendimiento educativo como criterio básico para determinar la calidad de la educación. El evaluador es un técnico altamente experimentado en la elaboración de instrumentos, para ejercer la práctica de una evaluación externa.

- La segunda generación corresponde a una evaluación externa que tiene su origen posteriormente a la Primera Guerra Mundial. Es una "evaluación descriptiva", que considera insuficiente el "rendimiento del estudiante" como único criterio de calidad. El evaluador continúa con su papel técnico, pero además pasa a describir el logro de objetivos.

- La tercera generación se denomina "de juicio", porque incluye el juicio de valor como parte fundamental del acto de evaluar. Esta posición agrega al evaluador un papel de juez, además de las funciones señaladas en las otras dos generaciones. El juicio de valor 
corresponde a una interpretación particular ofrecida sobre la base de estándares de comparación, los cuales se valoran dentro de una connotación científica.

Desde el punto de vista de evaluación curricular, esta tercera generación evidencia claramente un "enfoque tecnológico". Críticamente, de Alba, afirma que bajo este enfoque se...

(...) pretende validar y legitimar las decisiones que se toman en relación a la evaluación, o sea que detrás del pretendido carácter científico de la evaluación se esconde una necesidad de quienes postulan el discurso, de legitimar las prácticas educativas, necesidad que va cobrando una fuerza cada vez mayor (de Alba, 1991, p.81).

Se implica, por tanto, que dentro de esta generación se ubica la autoevaluación para la acreditación. Según Dobles (1996), la acreditación surge en el contexto estadounidense norteamericano, a principios del siglo XX. Es así como a partir de la acreditación de escuelas secundarias, se desprende la acreditación universitaria.

Tradicionalmente se considera que la acreditación está basada en una relación vertical, entre un organismo externo (la comisión acreditadora), como ente activo que preestablece estándares y criterios; y un ente interno (el programa o la institución), con un papel más pasivo, que se apega a las reglas establecidas y realiza un autoestudio tratando de complacer las demandas del organismo acreditador (Dobles, 1996).

- La cuarta generación, denominada como "constructiva", responde a la posición epistemológica desde la cual se realiza. También se le llama interpretativa o sensible, en el sentido de poder diseñar diferentes formas de enfocar una evaluación. De tal manera, los límites y parámetros se construyen conjuntamente, entre las personas involucradas y los evaluadores, razón por la cual también se le denomina "evaluación emergente" (Guba y (Lincoln, 1989).

Según Dobles (1996), la evolución de la autoevaluación y acreditación, como procesos evaluativos, implican a su vez, una generación de cambio en la visión social educativa y en la visión de cómo se construye el conocimiento humano y del para qué se desea la autoevaluación. Según la autora, se da el surgimiento de un paradigma alternativo, donde el acreditado pasa de estar sometido a lo que el evaluador desea, con poca reflexión, a tener un papel más activo, por cuanto se convierte en constructor y reconstructor de su propia realidad. 
Bajo la perspectiva de una cuarta generación, Dobles (1996) señala que la acreditación deberá otorgar tanta importancia al organismo externo como al interno, para lograr un acercamiento y una interacción, con el propósito de que, entre todas las personas, se construya un clima de reflexión del quehacer mismo, para encontrar soluciones creativas a los problemas. Esta situación, agrega dicha autora, conlleva un trastorno en el papel de la agencia acreditadora, que pasaría de ser un ente fiscalizador y controlador, a un ente involucrado plenamente con los participantes en el proceso, donde se adquieren compromisos morales.

Ahora bien, teniendo presentes estos puntos de vista, se observa con más claridad, la relación entre propósito y enfoque. La autoevaluación para el mejoramiento, sin otra pretensión, suele ser un proceso de convencimiento interno por parte de quienes desarrollan programas o planes de estudio. Se caracteriza además por presentar diseños y períodos de tiempo irrestrictos, con un riesgo potencial de no concretar acciones o no finalizar los procesos, sino existe un verdadero control metodológico. (Chacón, 2005)

Por otra parte, si la intención de la autoevaluación, principalmente, es la acreditación, entonces, aquella puede surgir, como resultado del convencimiento de las personas responsables de una carrera o programa; pero también puede corresponder al resultado del convencimiento de unos pocos, e incluso, de la decisión impuesta por una determinada persona. Desde esta perspectiva, el carácter acreditable de la autoevaluación la ubica esencialmente dentro de un enfoque tecnológico.

\section{Caracterización de un modelo de autoevaluación: un modelo fluctuante.}

Para la caracterización del modelo metodológico, como segundo objeto simultáneo en estudio, se utilizaron dos estrategias fundamentales: la sistematización y la reflexión constantes. Se determinaron además, para este segundo objeto de estudio, tres características: su presencia paralela al primer objeto de estudio, su visión particularizada (correspondiente al equipo investigador y, especialmente, al de la investigadora principal) y las estrategias no preescritas para su abordaje.

Dadas estas características, se entró en un plano epistémico de conocimiento del proceso mismo. A diferencia del primer objeto de estudio, que se rigió por criterios preestablecidos, este segundo objeto se visualizó metodológicamente más flexible, aunque no fácil de sistematizar ni configurar, dado lo ambicioso del proyecto, por cuanto se autoevaluaron varios planes de estudio de manera simultánea. En su complejidad, se distinguieron tres fases para la caracterización del proceso metodológico. 
- Fase de acercamiento conceptual: cómo entender el proceso mismo como objeto de estudio.

- Fase de reflexión asistemática: un cuerpo de reflexiones asistemáticas. Es la fase que da el insumo para las siguientes.

- Fase de distanciamiento: toma de distancia del proceso para clarificar el cuerpo axiológico propio de las investigadoras, como elemento influyente.

- Fase de concreción: configuración del proceso metodológico de la autoevaluación, desde el punto de vista investigativo.

Ahora bien, para lograr la caracterización del modelo de autoevaluación se consideran los siguientes aspectos:

- $\quad$ El contexto: La Escuela de Formación Docente.

- La identificación e interpretación de características paradigmáticas investigativas.

- Premisas investigativas del proceso de investigación.

- La intencionalidad de la autoevaluación.

- $\quad$ El papel del equipo investigador.

- El papel de las personas involucradas.

- $\quad$ El estilo del proceso metodológico.

- $\quad$ La organización de resultados.

\subsection{El contexto: la Escuela de Formación Docente.}

Esta unidad académica pertenece a la Facultad de Educación, fue creada en 1977, cuando asume los planes de estudio para la formación de docentes: Educación Secundaria, Educación Primaria y Educación Preescolar.

En la actualidad, esta unidad académica alberga un promedio de dos mil estudiantes, unos treinta docentes propietarios, de diversas especialidades; y otro tanto de docentes con carácter de interinazgo. Como tal, esta escuela está conformada por tres departamentos:

- El Departamento de Educación Primaria y Preescolar, que a su vez cuenta con dos secciones. La Sección de Educación Preescolar administra el plan de estudio de Bachillerato y Licenciatura en Educación Preescolar. Por otra parte, la Sección de 
Educación Primaria administra el plan de estudio de Bachillerato y Licenciatura en Educación Primaria.

- El Departamento de Educación Secundaria, comparte diez planes de estudio con otras unidades académicas: Enseñanza del Inglés, Enseñanza del Francés, Enseñanza del Castellano y Literatura, Enseñanza de los Estudios Sociales, Enseñanza de las Ciencias, Enseñanza de la Matemática y la Física, Enseñanza de la Música, Enseñanza de las Artes Plásticas, Enseñanza de la Filosofía, Enseñanza de la Psicología. Todas estas carreras, a excepción de Artes Plásticas y Música, cuentan con una salida lateral que otorga el título de profesorado en la Enseñanza de la Especialidad al tercer año aprobado de carrera.

Este departamento administra el llamado Núcleo Pedagógico que comparten estas diez carreras.

Según la normativa, las carreras compartidas se administran en conjunto con otras unidades académicas; sin embargo, es la Escuela de Formación Docente la "unidad académica base". La figura de "comisiones interdiciplinarias" nace para garantizar la participación equitativa de las diferentes unidades académicas involucradas en los planes de formación. (Chacón, 2004, p. 100)

Aunque existe la figura de "comisiones compartidas", éstas no tienen ingerencia directa sobre la gestión del Núcleo Pedagógico. Esta gestión corresponde al Departamento de Educación Secundaria. (Chacón, 2004, p. 101)

Se hace necesario agregar aquí las definiciones sobre Núcleo Pedagógico y Tronco Común. El "Informe de Investigación Evaluativa: Autoevaluación de la Escuela de Formación Docente", señala que el Núcleo Pedagógico:

Corresponde al componente pedagógico organizado que comparten los 10 planes de estudio del Departamento de Secundaria. Este Núcleo Pedagógico está fundamentado sobre unos objetivos y principios y que dan sustento a un conjunto de 40 cursos integrados por: un Tronco Común de la Escuela de Formación Docente, un Tramo Diferenciado, un Bloque Compartido de cursos de servicio y un curso optativo de educación. Para cada plan de estudio compartido el Núcleo Pedagógico representa 13 cursos (...) (Chacón, 2004, p. 101)

Por su parte, el mismo informe indica que el Tronco Común de la Escuela de Formación Docente: 
Corresponde al grupo de cursos de fundamentos básicos educativos, que son compartidos por los planes de estudio, en el nivel de bachillerato, de la Escuela de Formación Docente: Educación Preescolar, Educación Primaria y Enseñanza Secundaria. En la actualidad estos cursos son administrados por el Departamento de Secundaria y corresponden a los siguientes 5 cursos (...) (Chacón, 2004, p. 101)

- El Departamento de Docencia Universitaria se dedica a la capacitación de docentes en cuanto a la pedagogía universitaria y no cuenta con ningún plan de estudio.

En concreto, la Escuela de Formación Docente representa una compleja organización administrativo-curricular, cuya máxima expresión se da en las asambleas de escuela, donde se confluyen las más diversas opiniones, con momentos de confrontación y de consenso.

\subsection{Identificación de características paradigmáticas investigativas.}

La ejecución del modelo de autoevaluación fluyó entre el paradigma naturalista y positivista, con características propias de un enfoque cualitativo y cuantitativo. Para su configuración se utiliza una serie de características distintivas, según cada enfoque, con limitaciones en ambos, según se observa en el siguiente cuadro: 


\section{El modelo de autoevaluación utilizado según las características investigativas.}

\begin{abstract}
Características y análisis de la investigación, a la luz de los aspectos cualitativas, según Dobles y otras, (1996)

1. Su propósito es comprender, interpretar y explicar la realidad para transformarla.

- El conocimiento de las investigadoras es de carácter hermeneúticodialéctico. Es individual y personal en una primera etapa, para trasformarse, finalmente, en una construcción colectiva. Los valores inciden en la investigación y forman parte de la realidad-realidades.

\section{APLICACIÓN:}

La autoevaluación busca como propósito comprender e interpretar para mejorar el desarrollo de los planes de estudio. En una primera etapa el conocimiento fue personal para el equipo investigador $y$, posteriormente, se dio una construcción colectiva, para entender una realidad holística pero particular, y para sugerir estrategias de mejoramiento.

2. La teoría constituye una reflexión que se da desde la praxis.

- La realidad no sólo se constituye de los hechos observables, sino también por los significados, símbolos e interpretaciones elaboradas por las y los sujetos mediante su interacción con los demás. La teoría hermeneútica se centra en la identificación y comprensión de las reglas que subyacen, siguen y gobiernan los fenómenos sociales. Se buscan patrones de intercambio que son el resultado de compartir significados e interpretaciones sobre las realidades.
\end{abstract}

\section{APLICACIÓN}

Según lo observado y la información obtenida, se establecen interpretaciones y reflexiones que surgieron desde diversos ámbitos: el equipo investigador, el cuerpo de docentes, las direcciones de departamentos, para interactuar en diversos espacios

3. Describe, explica e interpreta la situación en la que se desarrolla el acontecimiento.

En primera instancia, se producen datos descriptivos: las propias palabras de las personas habladas o escritas y los diferentes aspectos que se han observado. Sobre esta base, se buscó comprender, explicar e interpretar la situación en su contexto.
Cánones científicos desde la tradición positivista para la investigación cualitativa, según Pinto, (1996)

1. La confiabilidad externa e interna está dada por la rigurosidad en el diseño y aplicación de instrumentos de recolección de datos.

Se exigen varios observadores y repetidas observaciones, entrevistas reiteradas grabadas de manera mecánica. Se exige definir con precisión las categorías para la obtención y codificación de los datos.

\section{APLICACIÓN}

El equipo investigador asumió los espacios de aplicación de instrumentos o plenarias de manera conjunta, al menos dos personas permanecieron en los diferentes momentos. Las plenarias fueron grabadas y transcritas. Las preguntas abiertas fueron transcritas fielmente.

2. La validez externa e interna de la investigación, en cuanto a la precisión de las conclusiones y su replicabilidad en casos similares.

Se acepta la recopilación de matices de los hechos estudiados, así como comprender contextualmente tales hechos, para lograr conclusiones "totalistas", las que deben ser trianguladas con los datos recogidos. La validez está dada por la repetición del proceso indagativo en sitios múltiples.

APLICACION

Las interpretaciones están acordes en su mayoría a criterios preestablecidos, cuyos proceso indagativo fue repetido, especialmente con las tres poblaciones estudiantiles: preescolar primaria y secundaria

3. La incidencia práctica de los hallazgos para determinar políticas que puedan afectar significativamente a grupos humanos más amplios.

Se consideran positivos los hallazgos cualitativos que potencien o muestren relaciones de causales de ciertos problemas 


\section{Características y análisis de la investigación, a la luz de los aspectos} cualitativas, según Dobles y otras, (1996)

- Se requirió una rigurosa descripción contextual de un hecho o una situación para garantizar la máxima intersubjetividad en la captación de una realidad compleja. Recolección sistemática de datos.

\section{APLICACIÓN}

La descripción contextual es amplia, sin embargo, se ofrece para comprender curricular y administrativamente, los planes de estudio de Educación Preescolar, Educación Primaria y el Núcleo Pedagógica, se explica sobre su organización estructural y administrativa básicamente, no se llega a descripciones de lo cotidiano.

La recopilación de la información fue sistemática.

\section{Profundiza en los diferentes aspectos que influyen sobre un problema,} situación o fenómeno.

- La realidad es holística, global y polifacética, nunca estática; se transforma y se crea, por tanto, tiene una estructura intrínsicamente significativa, constituida y sostenida por las acciones interpretativas de sus miembros. Por eso existen muchas realidades y no una sola.

\section{APLICACIÓN}

No se profundiza, se dan valoraciones especificas, pero no profundas. Sólo en algunos aspectos tales como la relación de plazas en propiedad e interinos, sí se ofrece un mayor análisis.

5. La identidad personal, socio-cultural y profesional de la o el investigador, influye en su forma de observar, comprender y actuar en el mundo.

- En la evaluación los investigadores llevan un cuerpo axiológico de influencia: sus sentimientos creencias, valores, prejuicios que influyen también en los datos.
Cánones cientificos desde la tradición positivista para la investigación cualitativa, según Pinto, (1996)

\section{APLICACIÓN}

Los hallazgos tienen una consecuencia esencial y el logro de líneas de autorregulación con miras a la acreditación, afectan todo el quehacer en el desarrollo de los planes de estudio y las personas involucradas.

Se dan algunas relaciones de causa y efecto, pero también se ofrecen relaciones multifactoriales o simplemente, se ofrecen descripciones.

4. La neutralidad del investigador en el tratamiento y la explicación de la información y el conocimiento del fenómeno indagado.

Neutralidad entendida como el intento de que los resultados no estén influenciados por las motivaciones, intereses e inclinaciones del investigador. Se hace necesaria la confirmación mediante los datos producidos.

\section{APLICACIÓN}

El grupo investigador es parte de la unidad académica, desde esta perspectiva evitar las motivaciones o intereses fue difícil. La motivación más bien fue vista como un elemento a favor en el sentido de mantener vivo un interés en el proceso.

Se utilizaron tres estrategias para evitar sesgos:

- Interpretar sobre la base de información

- Cada investigadora se hizo responsable de aquel plan de estudio que menos conocía, aunque todas tenían niveles de conocimiento de los tres planes.

- Reflexión individual y conjunta, resepcto a los resultados, los procedimientos, sus consecuencias y sentimientos.

5. Enunciar principios explicativos y predictivos sobre la realidad estudiada.

Es necesario rescatar los conceptos esenciales del fenómeno estudiado para elaborar abstracciones teóricas que expliquen e interpreten, en general, hechos similares. APLICACIÓN 


\section{Características y análisis de la investigación, a la luz de los aspectos} cualitativas, según Dobles y otras, (1996)

La posición de las investigadoras en el proceso autoevaluación fue implicativa, afectiva, estructural y, profesionalmente.

- Afectivamente: porque las tres contaban con muchos años de laborar en la unidad académica; conocían la cultura y mantenían vínculos afectivos con las personas. Existían preconcepciones.

- Estructuralmente: por tener un papel docente, pero también como directoras de departamento (durante el primer año de ejecutada la investigación).

- Profesionalmente: porque los mismos resultados rozaban el propio quehacer académico.

Ahora bien, para la totalidad de los participantes, en el cuerpo docente, la implicación profesional siempre estuvo presente, pero también la afectiva. Se dieron reacciones abiertas frente al proceso de autoevaluación, algunos para avalar el proceso, otros para criticarlos, o para reaccionar ante los resultados logrados.

La Asociación de Estudiantes tuvo un nivel de implicación afectivo y estructural.

6. La persona es un sujeto interactivo, comunicativo que comparte significados.

- La relación sujeto-problema es interactiva. El sujeto necesita leer las situaciones concretas, interpretativas y recurrir a su experiencia práctica. La persona construye su acción.

\section{APLICACIÓN}

Los participantes fueron comunicativos, para referirse esencialmente a tres aspectos: los resultados, el proceso metodológico seguido, el uso de la información. Incluso para manifestar posiciones radicales como no estar de acuerdo con el proceso metodológico.

\section{Enfoca integral y dialécticamente el tema de estudio.}

- La comprensión y la explicación juegan un papel fundamental en el círculo hermenéutico por lo que están en permanente interacción. En este proceso está siempre presente la analogía entre el texto escrito como expresión de un tipo de acción humana y la acción humana en general.

- La teoría se apoya en la interpretación de la realidad y es posible
Cánones científicos desde la tradición positivista para la investigación cualitativa, según Pinto, (1996)

Se ofrecen principios explicativos, pero muy enlazados a los factores y criterios preescritos. Sin embargo se logran rescatar dos producciones importantes:

La concepción sobre demandas y necesidades a las que debe responder la formación de docentes de la unidad académica. Este fue un proceso consensuado por parte de todos los docentes de la unidad académica que participaron.

La propia caracterización del modelo metodológico utilizado.

\section{El valor de verdad de los descubrimientos.}

Establecer confianza en la verdad de los descubrimientos, por medio de la confrontación de las creencias e interpretaciones del investigador con las creencias e interpretaciones de otras fuentes. Entre éstas últimas, las más importantes son la de los participantes del hecho estudiado. (Según Erazo, en Pinto, 1996)

\section{APLICACIÓN}

Se contrastan creencias de varias fuentes, pero no en todos los casos, puesto que no en todos los aspectos era aplicable la confrontación, aquellos en que la información era básicamente descriptiva de la existencia por ejemplo "de normativa", o porque el procedimiento en la Guía de Evaluación no lo solicitaba. 


\begin{tabular}{|c|c|}
\hline $\begin{array}{l}\text { Características y análisis de la investigación, a la luz de los aspectos } \\
\text { cualitativas, según Dobles y otras, (1996) }\end{array}$ & $\begin{array}{l}\text { Cánones científicos desde la tradición positivista para la } \\
\text { investigación cualitativa, según Pinto, (1996) }\end{array}$ \\
\hline $\begin{array}{l}\text { comprenderla y explicarla a partir del análisis estructural de sus } \\
\text { dimensiones y de lo que forma parte. }\end{array}$ & \\
\hline $\begin{array}{l}\text { APLICACIÓN } \\
\text { La interpretación fue sustentada con los datos alcanzados mediante técnicas } \\
\text { intencionadas, pero también, por el conocimiento del contexto que las } \\
\text { investigadoras tenian, por lo que se dieron supuestos para comprender } \\
\text { acciones. } \\
\text { No en todos los casos se tratan de dar explicaciones, en ocasiones } \\
\text { simplemente se aporta la información. }\end{array}$ & \\
\hline $\begin{array}{l}\text { 8. Explicita los tres niveles que la fundamentan y condicionan: el ontológico- } \\
\text { axiológico, el epistemológico y el metodológico. } \\
\text { - Es preciso explicitar los tres niveles en una propuesta. }\end{array}$ & \\
\hline $\begin{array}{l}\text { APLICACIÓN } \\
\text { Se explicitan en un documento aparte que no es posible aportar aquí, sin } \\
\text { embargo, en este cuadro se percibe la evidencia de los tres niveles, además } \\
\text { se hace referencia a estos en aspectos posteriores. }\end{array}$ & \\
\hline $\begin{array}{l}\text { 9. La cooperación de los sujetos actuantes en el análisis de los datos. } \\
\text { - La cooperación de los sujetos actuantes en el proceso de análisis, } \\
\text { interpretación y discusión de los resultados, es una condición insoslayable } \\
\text { en una investigación dentro del paradigma naturalista. La comprensión e } \\
\text { interpretación parten del conocimiento previo que las y los sujetos } \\
\text { actuantes tienen sobre la situación estudiada y sobre el contexto, así } \\
\text { como de las acciones subjetivas de ellos. }\end{array}$ & \\
\hline $\begin{array}{l}\text { APLICACIÓN } \\
\text { Los sujetos cooperaron en el aporte de información y análisis de la misma. } \\
\text { Sobre todo se dio una gran reflexión sobre los resultados para confirmar, } \\
\text { rechazar o proponer acciones de mejoramiento. } \\
\text { Por su parte, el equipo investigador también interpretó la información } \\
\text { utilizando como un elemento, el conocimiento sobre la cultura de la unidad } \\
\text { académica o el conocimiento sobre los procedimientos institucionales, o el } \\
\text { mismo conocimiento de los planes de estudio. } \\
\text { Sin embargo, muchas veces la información fue procesada, e inicialmente } \\
\text { interpretada, antes de ser llevada a los participantes }\end{array}$ & \\
\hline
\end{tabular}




\subsection{Interpretación sobre las características paradigmáticas investigativas.}

Aunque no se logra la exhaustividad en la interpretación por efectos de espacio, se pretende determinar a la luz de los sustentos teóricos investigativos, la manera en que fue abordado el proceso de autoevaluación como un modelo que utilizó, básicamente, criterios preescritos (Guía de autoevaluación. CEA, 1998), el nivel técnico fue explícito, no así el sustento teórico investigativo, aspecto que debió ser incorporado por el equipo responsable.

De esta manera, según aprecia la investigadora principal, quien suscribe $y$, de acuerdo con el sustento teórico y el análisis establecido en el apartado anterior, el modelo de autoevaluación utilizado para la Escuela de Formación Docente fue un modelo "fluctuantedialéctico", desde dos perspectivas:

- Una perspectiva macro de la investigativa, la cual se establece entre el paradigma naturalista y el paradigma positivista.

- Una perspectiva más específica, que se refiere al enfoque evaluativo utilizado.

La primera perspectiva, nos ubica, contrario a lo que pudieran señalar algunos teóricos o prácticos de la investigación, que el modelo investigativo utilizado medió en una posibilidad de fluctuación metodológica entre lo cualitativo y positivista (cuantitativo), con limitaciones en ambas líneas, en donde la posición cualitativa tuvo mayor presencia en la relación sujeto-objeto, el interés por el proceso para lograr esta caracterización, la conformación del equipo investigador, las descripciones, los análisis documentales, las valoraciones inherentes a toda evaluación, el logro en buena medida de un proceso pertinente, que partió de criterios preescritos a un proceso evolutivo de toma de compromiso del propio proceso, que evolucionó de uno centralizado a uno de participación amplia. Con el uso de técnicas cualitativas tales como:

- Análisis documentales variados, caracterizados por análisis de coherencia o congruencia, estos procedimientos fueron muy fuertes.

- Triangulaciones teóricas que parten de de diversas fuentes, con técnicas intertextuales y extratextuales y estableciendo relaciones entre conceptualizaciones y categorías establecidas.

- Entrevistas no estructuradas.

- Consultas delimitadas que solicitan información específica.

- Plenarias: con grupos de docentes, estudiantes, graduados, empleadores.

- Preguntas abiertas: establecidas como "comentarios", "observaciones"

- Establecimiento de categorías 
Por otra parte, la visión positivista tuvo mayor presencia en cuanto al interés por el resultado final; el alto nivel de dependencia respecto a elementos preescritos, no modificables: factores, criterios, indicadores; una guía de autoevaluación generalizable para cualquier proceso de autoevaluación institucional, un proceso inicial centralizado, el uso de técnicas cuantitativas, tales como: cuestionarios de respuesta cerrada, utilización de escalas preestablecidas: de frecuencia, de satisfacción, de calidad, escalas numéricas, tabulación y tratamiento estadístico de los datos.

Esta situación se complementa con el enfoque evaluativo utilizado, que igualmente fue fluctuante, entre un enfoque "tecnológico" y un enfoque de "autogestión (dialéctico)"

El enfoque tecnológico tiene presencia en los resultados y los aspectos preescritos ya mencionados: factores, criterios, indicadores, la guía de autoevaluación, pero más allá y de mayor impacto aún, en la intencionalidad del resultado, como bien fue aprobado en asamblea de escuela el "proceso de autoevaluación con miras a la acreditación", donde la acreditación representa una necesidad de legitimar una calidad y controlar. De esta forma, el equipo investigador respondió al mandato de la Asamblea de Escuela, aunque no e manera acrítica.

Durante el proceso, en lo interno, el equipo en repetidas ocasiones, objetó y reflexionó, respecto a diversos aspectos del proceso, ya fuera por convencimiento propio o por las repetidas observaciones señaladas por varios participantes.

El presente análisis, es un modelo de autogestión evaluativo que busca comprender y valorar una situación particular de una estructura o programa, que conlleva premisas varias.

\subsection{Premisas investigativas del proceso de investigación}

De acuerdo con lo anterior, se desprenden al menos, las siguientes premisas :

- Epistemológica: el conocimiento se produce de adentro hacia fuera. Aunque se logra un conocimiento particular, también se llega a un conocimiento general. Es decir, el conocimiento parte, en algunos momentos, de las diversas situaciones de cada plan de estudio. Pero por otra parte, se comprenden situaciones particulares de cada plan de estudio, por la dinámica "macro" de carácter institucional

- Ontológica: existen muchas realidades de influencia, dinámicamente cambiantes, incluyendo la realidad de las investigadoras. Pero también se presenta una realidad total que se debe que visualizar: la unidad académica y la realidad institucional. 
- Heurísticas: Es posible establecer relaciones de causa y efecto, pero también es factible establecer relaciones multifactoriales para la comprensión de la estructura.

- Axiológicas: por inherencia, esta premisa es esencialmente cualitativa, por cuanto la evaluación por sí busca la valoración fundamentada, en el procedimiento y lo objetivo como subjetividad conjunta, pero además la objetividad, en algunos aspectos, se logra por los datos estadísticos. Por este motivo, el proceso metodológico de la investigación y las valoraciones generadas, fueron mediadas por lo objetivo y subjetivo.

\subsection{La intencionalidad de la autoevaluación:}

La intención básica fue de corte evaluativo, cualitativo. Se trató de comprender la realidad o realidades para una valoración.

La aplicación de los resultados fue de corte tecnológico o autogestionario. La acreditación es una aplicación tecnológica que busca legitimar y controlar una situación y la perspectiva autogestionaria, permanece en el nivel interno, con el fin de mejorar, sin legitimación.

\subsection{El papel del equipo investigador:}

Estuvo conformado por tres personas, según el criterio de la dirección de Escuela de Formación Docente, y matizó su trabajo con el conocimiento previo de la realidad de la Escuela. Manejó dos papeles simultáneos; el primero, constituirse como "Comisión de Autoevaluación de la Escuela de Formación Docente"; el segundo, conformar el "Equipo Investigador del Proceso de Autoevaluación de la Escuela de Formación Docente" ante el Instituto de Investigación en Educación. Inicialmente este equipo manifestó un papel centralizado, asesorado con personal del Centro de Evaluación Académica de la misma universidad. Este equipo desarrolló las siguientes funciones:

- Elaborar el proyecto de investigación evaluativo.

- Organizrar y ejecución de un plan operativo.

- Mantener informados a los docentes y estudiantes sobre el proyecto, procesos, resultados.

- Sistematizar del proceso y los resultados. Esta función recayó básicamente en la investigadora principal.

- Descentralizar el proceso para una toma de posesión por parte de los docentes y para la toma de decisiones en cuanto a medidas autorregulatorias. 
La gestión del proyecto investigación estuvo a cargo de la investigadora principal, quien fungió como coordinadora de la Comisión de Autoevaluación.

\subsection{El papel de las personas involucradas.}

Estas personas fueron docentes, estudiantes y personas con puestos docenteadministrativos. Su papel se transformó de colaboradores a participaticipantes, comprometidos con la intención de mejorar la docencia.

Inicialmente fueron colaboradores para avalar el proyecto, otorgar información y socializar resultados. Posteriormente, conforme avanzaba el proceso, lograron un compromiso participativo que se concretó en la comprensión de los resultados de la autoevaluación, así como la toma de medidas de autorregulación configuradas en un plan.

\subsection{Estilo del proceso metodológico}

Se ofrece una autoevaluación democrática que comprende los siguientes aspectos:

- Las investigadoras reconocieron el valor pluralista de los valores. Esta premisa se evidenció por la presencia de tantos actores, que mantienen variadas realidades e ideologías, expresadas, en ocasiones, de manera convergente y en otras, de manera divergente.

- El valor básico es una comunidad informada. Aunque surgieron dos limitantes, se dieron varias vías de información, consideradas por algunos actores como suficientes y por otros, insuficientes. De igual manera, algunas personas se mostraron siempre interesadas y otras, por el contrario, no mostraron interés en informarse.

- El equipo investigador o coordinador fue accesible. Fue mediatizador con los diferentes grupos, sectores o sujetos, aunque algunos docentes siempre exigieron más acceso y mayor mediatización.

- Interesa la comprensión del programa. Se buscó la comprensión de lo planes de estudio y la unidad académica, aunque con un carácter reflexivo y crítico. Por efectos de tiempo el estudio del Núcleo Pedagógico fue insuficiente, y por tanto, su comprensión fue limitada.

- Un proceso metodológico pertinente, acorde con la cultura y dinámica de las personas involucradas.

El modelo ejecutado estuvo orientado por metas ambiciosas para el tiempo establecido, debido a la diversidad de ámbitos por estudiar y una comisión de autoevaluación 
de reducido número de integrantes y escasa carga académica. Con esta limitación, se estableció un proceso de gestión metodológica evolutivo que mostró tres fases:

- Fase inicial centralizada: el equipo propuso un proyecto y un plan operativo que fueron aceptados por la Asamblea de Escuela, con sugerencias de cambios o aclaraciones sobre dudas. En esta fase se clarifica la intencionalidad de la autoevaluación.

- Fase de coparticipación: El equipo responsable compartió información sobre resultados, ante lo cual, los diferentes participantes solicitaron aclaraciones; ofrecieron información y elaboraron propuestas de mejoramiento de estrategias de procedimiento, pero sobre todo, propusieron estrategias de mejoramiento de los planes de estudio. La participación fue colaborativa.

- Fase de participación comprometida: Los mismos grupos formularon estrategias de acción de mejoramiento y la toma de decisiones de procedimientos por seguir, ya fuera para continuar el proceso, detenerse, reiniciar; retomar parcialmente algunos aspectos; decidir sobre la pertinencia de la acreditación, entre otros aspectos. Se puede afirmar que esta fase fue de implicación.

De esta forma se observa un proceso complejo, por la cantidad de sujetos involucrados, y la variedad de aspectos por considerar, pero que ofrece resultados concretos que podrán ser utilizados para el mejoramiento del quehacer académico.

\subsection{Organización de resultados.}

La organización de resultados se caracterizó por el alcance de resultados comunes o convergentes y resultados diferenciados.

Los resultados diferenciados se establecieron por las características particulares de cada plan de estudio: Educación preescolar, Educación Primaria y al tramo de carrera ya mencionado "Núcleo Pedagógico de la Educación Secundaria". Los resultados convergentes tuvieron como elementos sobresalientes, el estudio del Tronco Común y el tramo de carrera ya señalado.

Los resultados diferenciados se organizaron en tres informes: 
- Informe de autoevalución del Bachillerato en Educación Preescolar. Este informe sirve de insumo para la organización del Informe de Autoevaluación con fines de acreditación ante el Sistema Nacional de Acreditación.

- Informe de autoevalución del Bachillerato en Educación Primaria. Este informe, de igual manera, sirve dee insumo para la organización del Informe de Autoevaluación con fines de acreditación ante el Sistema Nacional de Acreditación.

- Informe de autoevaluación del Núcleo Pedagógico para las Enseñanzas en Secundaria.

Estos informes presentan los resultados por factores, mencionados al inicio de este artículo: Plan de estudio, docencia, personal académico, población estudiantil, organización y administración; y recursos financieros, físicos y materiales.

Los resultados comunes y diferenciados se reúnen en el "Informe de Investigación Evaluativa: Autoevaluación de la Escuela de Formación Docente". Este informe abarca 440 páginas, sin incluir anexos, donde se pueden apreciar los resultados, tanto de los planes de estudio de Educación Preescolar, Educación Primaria, como del Núcleo Pedagógico. Este informe puede ser consultado en el Instituto de Investigación en Educación de la Universidad de Costa Rica.

La riqueza de este informe integrado reside en varios aspectos, una ampliación del análisis de los resultados de los informes diferenciados, una puntal descripción de cada plan de estudio, un marco de referencia que da sustento a la investigación y la incorporación de una sistematización metodológica detallada, la cual será motivo posteriormente, para presentar un tercer artículo. 


\section{CONSIDERACIONES FINALES}

El proceso de autoevaluación en la Escuela de Formación Docente, resultó una labor ardua y larga que demandó un esfuerzo, mucho más allá de la carga asignada y del esfuerzo profesional del equipo investigador.

Abordar el proceso de manera investigativa, demandó un estudio y análisis de dos objetos de estudio: la intención de la autoevaluación y cómo se realiza, para determinar un acercamiento paradigmático que permitiera finalmente, tomar conciencia del papel del investigador y de los involucrados. Este análisis también permitió generar una caracterización del modelo de autoevaluación, que, aunque no exhaustivo, si brinda elementos para determinar de qué manera tienen presencia, tanto los sujetos, como las investigadoras y las premisas sobre las que se sustenta la investigación.

Metodológicamente el proceso implicó un abordaje complejo, al ser asumido por una sola comisión para tres carreras, situación particular en la Universidad de Costa Rica. Se recomienda en un futuro integrar comisiones diferenciadas, de manera más equitativa, con asignación de una carga real.

La organización de resultados remite a los documentos de sumo interés, especialmente el informe integrado de resultados, ya que reúne las condiciones propias de una investigación, pero a la vez, los insumos necesarios para posteriores informes con fines de acreditación.

Por otra parte, la mayor pretensión en el futuro, para una autoevaluación más pertinente en un contexto mulficatorial, sería trascender de una autoevaluación de tercera generación (controladora y preescrita), a una de cuarta generación (emergente, más democrática e implicativa). Esta transición podrá hacer surgir una serie de procedimientos metodológicos diversos, híbridos entre enfoques y paradigmas. Pero si realmente se desea llegar a una cuarta generación de la autoevaluación, se necesitará, por parte de quienes se responsabilizan de ella, ya sean investigadores, evaluadores, comisiones o grupos, de una toma de conciencia clara y transparente, respecto al papel que están asumiendo, a la razón de ser de la autoevaluación y al cómo se llega al conocimiento de la realidad.

La toma de conciencia permitirá a la autoevaluación, tomar lugar bajo un enfoque "constructivo" y bajo un punto de vista "contextual o axiológico", para considerarla dentro del ámbito de la investigación, sin embargo, será necesario madurar los procesos, de tal manera que sea el momento histórico el que ofrezca las condiciones para que dicha evolución se produzca. 
Ahora bien, desde el punto de vista investigativo, la presencia simultánea de los paradigmas cuantitativo y cualitativo, conlleva a determinar la necesidad, por parte de quienes asumen procesos similares, de contar con una conciencia clara de las intenciones de la autoevaluación. Cuya intención primera y fundamental, debe ser el mejoramiento de programas, mediante un proceso reflexivo que conduzca a desarrollar, entre otros aspectos, los siguientes:

- Sensibilidad en las personas involucradas, para aceptar la existencia de debilidades.

- Conocimiento de la realidad académico-profesional en la cual están inmersas las personas

- Clarificación del objetivo de estudio al cual se dedica la carrera o programa.

- Compromiso para asumir la responsabilidad "colectiva" de la organización y desarrollo de las carreras y sus planes de estudio.

- Compromiso colectivo para desarrollar propuestas de mejoramiento que impliquen acciones, a nivel individual, grupal, e institucional.

- Comprensión de la cultura evaluativa como característica permanente que identifica a las instancias gestoras de carreras o programas.

- Comprensión del acto de acreditación como una acción institucional relacionada a lo externo, que da fe de una calidad mínima y que puede o no tener repercusiones sociales. Pero que de ninguna manera garantiza de manera permanente e indefinida, la calidad, ya sea de mínimo o alto nivel, de una carrera o programa.

De tal manera que, en educación superior, particularmente en la universidad pública, la autoevaluación como práctica permanente, debe convertirse en una cultura vinculada a la sociedad. Pero que conlleve a la reflexión, en todos sus niveles estructurales, de tal manera que permita determinar con claridad, las repercusiones de la acreditación.

Adicionalmente, al considerar que el plano reflexivo no es suficiente, la universidad pública está obligada a buscar las condiciones para que los procesos de autoevaluación y particularmente el acto de acreditación, guarden un balance coherente entre el esfuerzo humano, el costo económico y los beneficios que de éstos se derivan, salvaguardando en todo momento la autonomía universitaria. 


\section{REFERENCIAS.}

Chacón, S. (2004). Informe de Investigación Evaluativa: Autoevaluación en la Escuela de Formación Docente. San José, Costa Rica: Instituto de Investigación de la Educación, Universidad de Costa Rica.

Chacón, S (2005). Acercamiento paradigmático de la Autoevaluación como investigación. En Revista Electrónica Actualidades Investigativas en Educación. 5(1). Disponible en http://revista.inie.ucr.ac.cr

Dobles, M. C.; Zúñiga, M., García, J. (1996). Investigación en educación: procesos interacciones construcciones. Costa Rica: EUNED.

Dobles, C. (1996). Acreditación en Educación Superior: Orígenes, prácticas actuales, perspectivas. En Memoria Foro Taller Internacional sobre Tendencias Actuales en la Medición y Evaluación Educativa. Compiladora Leda Badilla. Universidad de Costa Rica.

Guba, E. y Lincoln, Y. (1989). Fourth Generation Evaluation. California: SAGE Pub. Incorporated.

Pinto, R. (1996). Algunos Criterios de Calidad Científica de la Investigación Cualitativa en Educación. En Memoria Foro Taller Internacional sobre Tendencias Actuales en la Medición y Evaluación Educativa. Compiladora Leda Badilla. Universidad de Costa Rica.

De Alba, A. (1991). Evaluación Curricular. Conformación conceptual del campo. México: Centro de Estudios sobre la Universidad de México.

Universidad de Costa Rica. (1998). Guía de Autoevaluación. San José, Costa Rica: Centro de Evaluación Académica. Universidad de Costa Rica. 